\title{
Deterministic phase transitions and self-organization in logistic cellular automata
}

\author{
M. Ibrahimi $\odot,{ }^{1}$ O. Gulseren $\odot,{ }^{1,2, *}$ and S. Jahangirov $\odot^{2,3, \dagger}$ \\ ${ }^{1}$ Department of Physics, Bilkent University, Ankara 06800, Turkey \\ ${ }^{2}$ UNAM-Institute of Materials Science and Nanotechnology, Bilkent University, Ankara 06800, Turkey \\ ${ }^{3}$ Interdisciplinary Program in Neuroscience, Bilkent University, Ankara 06800, Turkey
}

(Received 12 March 2019; published 25 October 2019)

\begin{abstract}
We present a simple extension in which a single parameter tunes the dynamics of cellular automata (CA) by consequently expanding their discrete state space into a Cantor set. Such an implementation serves as a potent platform for further investigation of several emergent phenomena, including deterministic phase transitions, pattern formation, autocatalysis, and self-organization. We first apply this approach to Conway's Game of Life and observe sudden changes in the asymptotic dynamics of the system accompanied by the emergence of complex propagators. Incorporation of the new state space with system features is used to explain the transitions and formulate the tuning parameter range where the propagators adaptively survive by investigating their autocatalytic local interactions. Similar behavior is present when the same recipe is applied to Rule 90, an outer totalistic elementary one-dimensional cellular automaton. In addition, the latter case shows that deterministic transitions between classes of CA can be achieved by tuning a single parameter continuously.
\end{abstract}

DOI: 10.1103/PhysRevE.100.042216

\section{INTRODUCTION}

Cellular automata (CA), first invented to investigate selfreplication [1], are among the most frequently used tools to model and understand complex systems [2]. These computational frameworks are defined in discrete space-time-state domains, where time evolution occurs through local interactions. Despite the simple properties and the succinct absence of long-range connections, these implementations have been proven proper for studying large-scale collective behavior and self-organizing mechanisms, which often emerge in several dynamical systems including fluids [3], social [4] and urban [5] settings, or pattern formation in geological [6] and biological $[7,8]$ environments. By presenting a simple approach with two archetypal models, this work addresses critical deterministic phase transitions and tunable emanation of self-organized morphologies in such discrete domains.

One of the most studied CA, Conway's Game of Life (GOL), is an outer totalistic model of simple rules, defined in a two-dimensional square grid of cells and designed with two states, referred to as dead and alive, respectively. Despite the simplicity of the governing rules (described below), active states in Conway's GOL exhibit interesting dynamics, including stable, oscillating, and propagating structures akin to living systems. Asymptotic density and time evolution in GOL have been analyzed in detail using earlier theoretical approaches $[9,10]$. Generalizations of GOL include deterministic systems with variations in the rules [11] or expansion in the neighborhood [12] and stochastic systems involving probabilistic evolution [13,14] or asynchronous updating [15]. These generalizations are accompanied by additional tunable parameters, and they lead to the conclusion that GOL is a

\footnotetext{
*gulseren@fen.bilkent.edu.tr

†seymur@unam.bilkent.edu.tr
}

subcritical system dominated by quiescent states, but the same framework is still associated with critical transitions in its dynamics. The same conclusion is reached when GOL is compared to the so called set of Life-Like CA [16]. Scale-free properties of GOL have also been investigated in the context of self-organized criticality where the system is subject to slight external perturbations [17].

Subcriticality in GOL can be attributed to its limited state space, which results in a fast pace of decay and growth. Overcoming this subcriticality without sacrificing the deterministic nature of GOL not only redirects the system toward richer dynamics, but it also enables the exploration of additional phenomena that this framework may exhibit, including deterministic phase transitions and emergence in terms of pattern formation and self-organization.

In this paper, following the spirit of the well-known Logistic Map [18], which itself is a simple one-dimensional model of chaotic phase transitions, we introduce a single parameter that tunes the pace of decay and growth by consequently expanding the binary state space of CA into a Cantor set. As a result, we report a series of deterministic phase transitions that eventually lead to self-organized pattern formation. Initially, we observe a critical transition in density defining two main regimes: from sparse inactive to dense active asymptotic states. This is followed by other sharp transitions in density within the active asymptotic regime. Furthermore, we observe the tunable emanation and existence in a continuous range of a ballistic propagator that serves as a prototype showing how finite sets of local interactions get coupled to act as attractors in CA. Finally, we apply our framework to Rule 90, which is a one-dimensional elementary CA. This system not only shows a phenomenological behavior similar to the Logistic GOL, but it also enables parameter-tuned class transitions that can be used to investigate Langton's "computation at the edge of chaos" in detail [19]. 

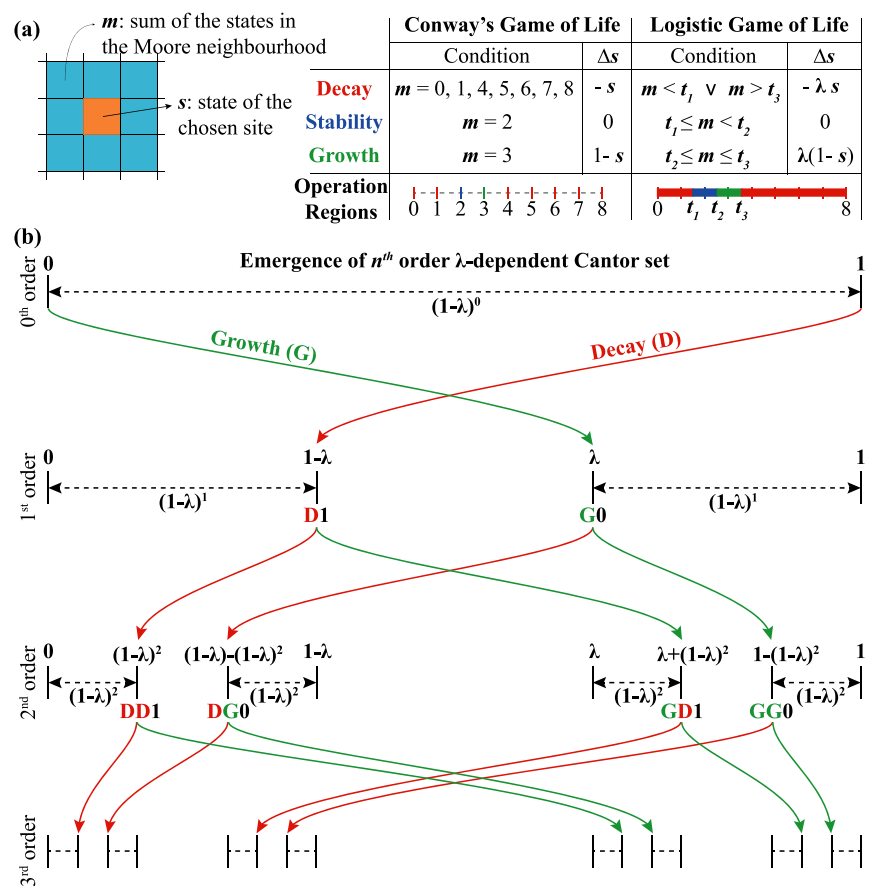

FIG. 1. (a) Conway's Game of Life (GOL) is defined in a square grid where the operation on each state $s$ is determined by the sum $m$ of the eight states in its Moore neighborhood. The table shows the conditions and difference equations for the three operation regimes: decay, stability, and growth. In the case of Logistic GOL, the difference equations are rescaled by the parameter $\lambda$. Operation regions are discrete in Conway's GOL while in Logistic GOL they are continuous domains separated by parameters $t_{1}, t_{2}$, and $t_{3}$. (b) Schematic representation of Cantor set formation starting with uniform distribution between 0 and 1 . The $n$ th-order Cantor set can be constructed by copying the $(n-1)$ th order and adding or subtracting $(1-\lambda)^{n}$. DG0 represents the element constructed by applying growth operation to 0 followed by decay operation.

The paper is organized as follows. In Sec. II, we introduce the Logistic extension of Conway's GOL. In Sec. III, by analyzing the asymptotic dynamics, we discuss a series of deterministic transitions occurring as the parameter is tuned. Section IV is devoted to developing a quantifiable formulation of the concept of autocatalytic interactions, and in Sec. V we discuss the emergence of a new propagator that is not present in Conway's GOL. In Sec. VI, we apply the same methodology to a one-dimensional system and observe class transitions. Finally, Sec. VII contains discussions of possible future directions.

\section{THE MODEL}

In Conway's GOL, each site, $s$, goes through a parallel updating scheme:

$$
s(\mathbf{r}, t+1)=s(\mathbf{r}, t)+\Delta s
$$

The change in a state, $\Delta s$, is a function of $s(\mathbf{r}, t)$ itself and $m(\mathbf{r}, t)$, which accounts for the sum of states in the site's Moore neighborhood, as shown in Fig. 1(a). In this automaton, $\Delta s$ can be defined by the rate equations that comply with three regimes: decay, stability, and growth, thereby offering a clear picture of the nonequilibrium conditions present in the system [see the table in Fig. 1(a)]. If $m<2$ or $m>3$, then $\Delta s=-s$, which in a two-state system indicates asymptotic decay. $m=2$ corresponds to stability, hence $\Delta s=0$. Finally, if $m=3$, then $\Delta s=1-s$, indicating asymptotic growth.

We extend GOL by introducing a single tuning parameter $\lambda$ that rescales the updating rate, with $\lambda=1$ corresponding to the original system. Actually these update equations are the same as the first-order finite-difference forms of exponential decay and asymptotic growth equations, respectively [see the table in Fig. 1(a)]. In the new system, sites are still subject to three possible rules, but with several consequences in the state space. We denote the updating rules as decay $(\mathbf{D})$, stability $(\mathbf{S})$, and growth $(\mathbf{G})$ operations:

$$
\mathbf{D} s \Rightarrow(1-\lambda) s, \quad \mathbf{S} s \Rightarrow s, \quad \mathbf{G} s \Rightarrow(1-\lambda) s+\lambda .
$$

For the range $0<\lambda<1$, the state space is eventually transformed into a Cantor set within the range $[0,1]$. The formation of this $\lambda$-dependent Cantor set is schematically shown in Fig. 1(b). If one starts with a continuous set between 0 and 1 , the decay and the growth operations will map these values to shrunken intervals in the limits $[0,1-\lambda]$ and $[\lambda, 1]$, respectively. Here both decay and growth operations map the states and increase the distribution density only within these smaller regions, while the stability operation has no such effect. After the first iteration, the range $[1-\lambda, \lambda]$ is still populated by values remaining the same due to the stability operation. However, they keep fading with subsequent iterations (sites that experience only stability in randomly initialized runs are statistically very rare), and hence they are not shown. The boundary values $\{0,1-\lambda, \lambda, 1\}$ formed after the first iteration are referred to as first-order elements of the Cantor set. Upon the second iteration, these boundaries are preserved while new boundaries are formed by the addition or subtraction of $(1-\lambda)^{2}$, leading to $\left\{(1-\lambda)^{2}, 1-\lambda-(1-\lambda)^{2}, \lambda+(1-\right.$ $\left.\lambda)^{2}, 1-(1-\lambda)^{2}\right\}$. Preserved and new boundaries together make up the second-order elements of the Cantor set. After $n$ iterations, one reaches at the $n$ th-order Cantor set elements $2^{n+1}$ unique values that are spaced in a self-similar fashion in the range $[0,1]$. Every element of the $n$ th-order Cantor set can be written as a sum $c_{0}+c_{1}(1-\lambda)^{1}+\cdots+c_{n-1}(1-$ $\lambda)^{n-1}+c_{n}(1-\lambda)^{n}$ with proper coefficients that take values $-1,0$, or 1 . Our preferred way of representing elements of the Cantor set is to write them as a series of decay and/or growth operated on 0 or 1 . As seen in Fig. 1(b), the order of polynomials representing a state is equal to the total number of decay and growth operations involved.

With the new state space we need to consider its effects on the neighborhood space $m$, which in turn dictates the rules. Now $m$ spans the range $[0,8]$ populated by eight-fold convolution of the Cantor set. To account for this, we fairly assign two unity intervals centered at 2 and 3 as stability and growth regimes, respectively, with the rest corresponding to decay. The limits of these intervals are represented by $t_{1}=$ $1.5, t_{2}=2.5$, and $t_{3}=3.5$, as shown in Fig. 1(a). The new automaton is named Logistic GOL and it is still synchronous, outer totalistic, and discrete in space and time, but now with an extended state space, so in terms of classification it can also be regarded as a coupled map lattice [20,21]. 


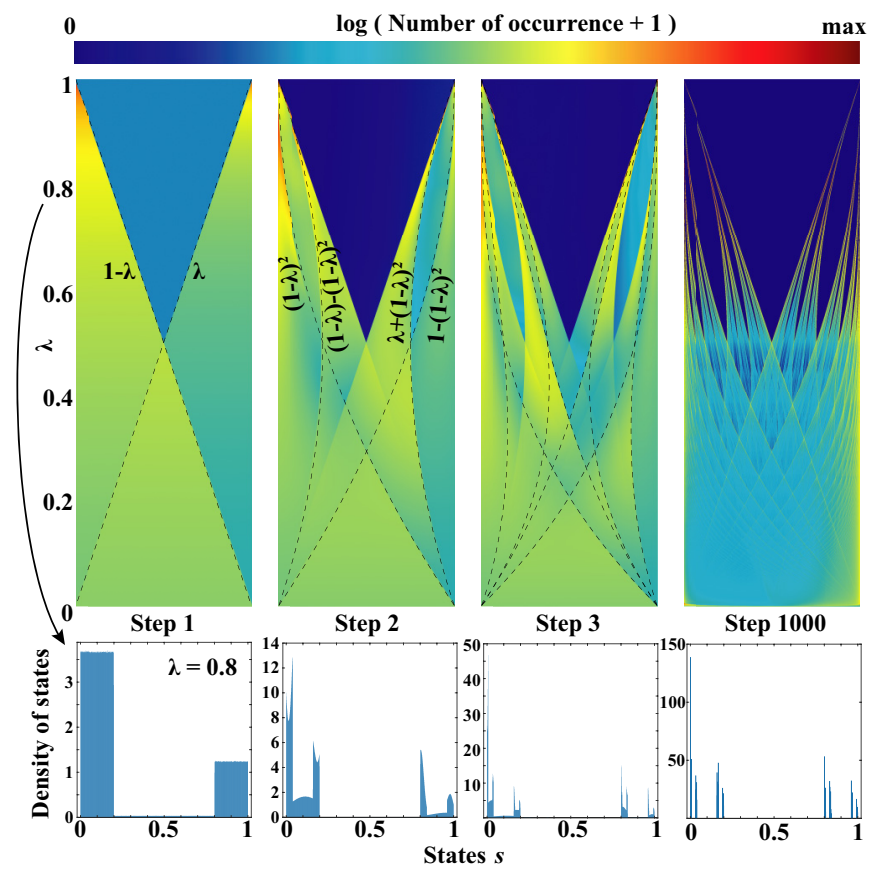

FIG. 2. Top panels: the logarithmic distribution of states after 1, 2, 3, and 1000 iterations. Two-dimensional histograms of states are constructed for values of $\lambda$ in the range $(0,1]$. The colors represent the logarithm of the number of occurrences plus unity, to avoid zeros (see the text). Dashed lines correspond to Cantor set elements formed after the corresponding iteration. Bottom panels: the density of states after $1,2,3$, and 1000 iterations for $\lambda=0.8$.

To further clarify how the Cantor set emerges in Logistic GOL, we perform numerical simulations in a $1000 \times 1000$ square lattice with periodic boundary conditions. Initially the states in each cell are picked up randomly from a uniform distribution in the range $[0,1]$. We start 3000 unique runs with different $\lambda$ linearly spanning the range $(0,1]$. After a certain number of iterations, we take a histogram that counts the number of occurrences of values that falls into one of the 1000 bins that uniformly parses the state space between 0 and 1 . The same is repeated 28 times with different initial conditions, and the histograms are summed over the repetitions. Finally, we take the logarithm of the number of occurrences after increasing each by 1 to avoid zeros. The resulting values depending on $\lambda$ and $s$ are represented with a color code that spans from 0 to an arbitrary maximum in a two-dimensional plot. The top panels of Fig. 2 show this analysis for steps 1, 2, 3 , and 1000 . One can clearly see the emergence of the first-, second-, and third-order Cantor set manifested as $\lambda$-dependent boundaries highlighted with dashed lines. After 1000 iterations, these boundaries remain while the regions in between fade away as described above. The bottom panels of Fig. 2 present the density of states for $\lambda=0.8$. Here the lack of the logarithmic scale further establishes the contrast between high- and low-density regions separated by the Cantor set.

\section{PHASE TRANSITIONS IN LOGISTIC GOL}

We now investigate Logistic GOL starting with $\lambda=1$ and tuning it down to see changes in the behavior of the system as it departs from Conway's GOL. In accordance with previous studies on GOL, we choose the asymptotic density, $\rho$, as our order parameter. To calculate $\rho$, we start with a $1000 \times 1000$ square lattice with periodic boundary conditions. Initially the states are picked up randomly from a uniform distribution in the range $[0,1]$ (initial density $\rho_{0}=0.5$ ). The system is first run for $10^{6}$ time steps for activity to settle and reach the thermodynamic limit. Then the system is run for another $10^{6}$ time steps for averaging the states over time and space. The same procedure is repeated 28 times for each value of $\lambda$ starting with different initial conditions, and the $\lambda$-dependent $\rho$ is found by taking the average of these runs.

The $\lambda$-dependent $\rho$ is shown in Fig. 3(a). Although Conway's GOL is well known for its long transients, its subcritical nature eventually leads it to a state dominated by the quiescent phase and sparsely distributed stable or periodic structures called still life and oscillators, respectively. We call this state the inactive asymptotic state. In the range $0.875<\lambda \leqslant 1$, Logistic GOL eventually reaches such an inactive asymptotic state. $\rho$ remains almost constant in this range, as shown in Fig. 3(a). Indeed, the only curious event that we encountered in this range was the fact that Gosper's Glider gun stops working at $\lambda \sim 0.896$.

At $\lambda=\lambda_{C}=0.875$ there is a critical transition in the system's behavior accompanied by a change in the average density. From this point on, the density starts increasing and the dynamics of the system keep showing qualitative and quantitative changes with respect to Conway's GOL, as seen in Fig. 3(a) and its insets. As $\lambda$ is decreased down to $\sim 0.8$, the dominance of the quiescent phase fades while the activity percolates. This leads to the emergence of chaotic regions consisting of rapidly changing disordered states, which we refer to as the flickering phase. After this point, the activity starts decreasing while the average density plateaus to a much higher value, $\rho \approx 0.44$. This value can in turn be justified by using an adapted single-site mean-field approximation $[9,10,16]$ over an initial density $\rho_{t}$ of uniform distribution:

$$
\rho_{t+1}=\rho_{t}+\lambda\left\{\rho_{t}\left[\int_{t_{1}}^{t_{2}} P(x) d x-1\right]+\int_{t_{2}}^{t_{3}} P(x) d x\right\},
$$

where $\rho_{t+1}$ is the updated density, and $P(x)$ is the neighborhood distribution obtained by the eightfold convolution of $\rho_{t}$ distribution. $\left[t_{1}, t_{2}\right)$ and $\left[t_{2}, t_{3}\right]$ are the neighborhood sum intervals defining the operation regimes of stability and growth, respectively. This equation has two $\lambda$ independent fixed points, the stable one being at $\rho_{t+1}=\rho_{t} \approx 0.45$.

Tuning $\lambda$ down further gives rise to domainlike structures of stable horizontal and vertical stripe patterns that interact with networks of flickering states at their boundaries. Similar stripe patterns were observed in the asynchronously updated version of GOL [15]. The flickering states can also propagate over the stripe patterns while changing them, allowing the system to comprise both ordered (stripes) and chaotic (flickers) phases in it simultaneously. It is important to notice that stripe patterns are actually a new dynamically stable configuration of the system. Dense sites self-organize in orthogonal orientations, and each of them neighbors only two other dense sites by remaining in the stability regime $\left(t_{1}<m<t_{2}\right)$, whereas the surrounding empty sites that actually make up 
(a)
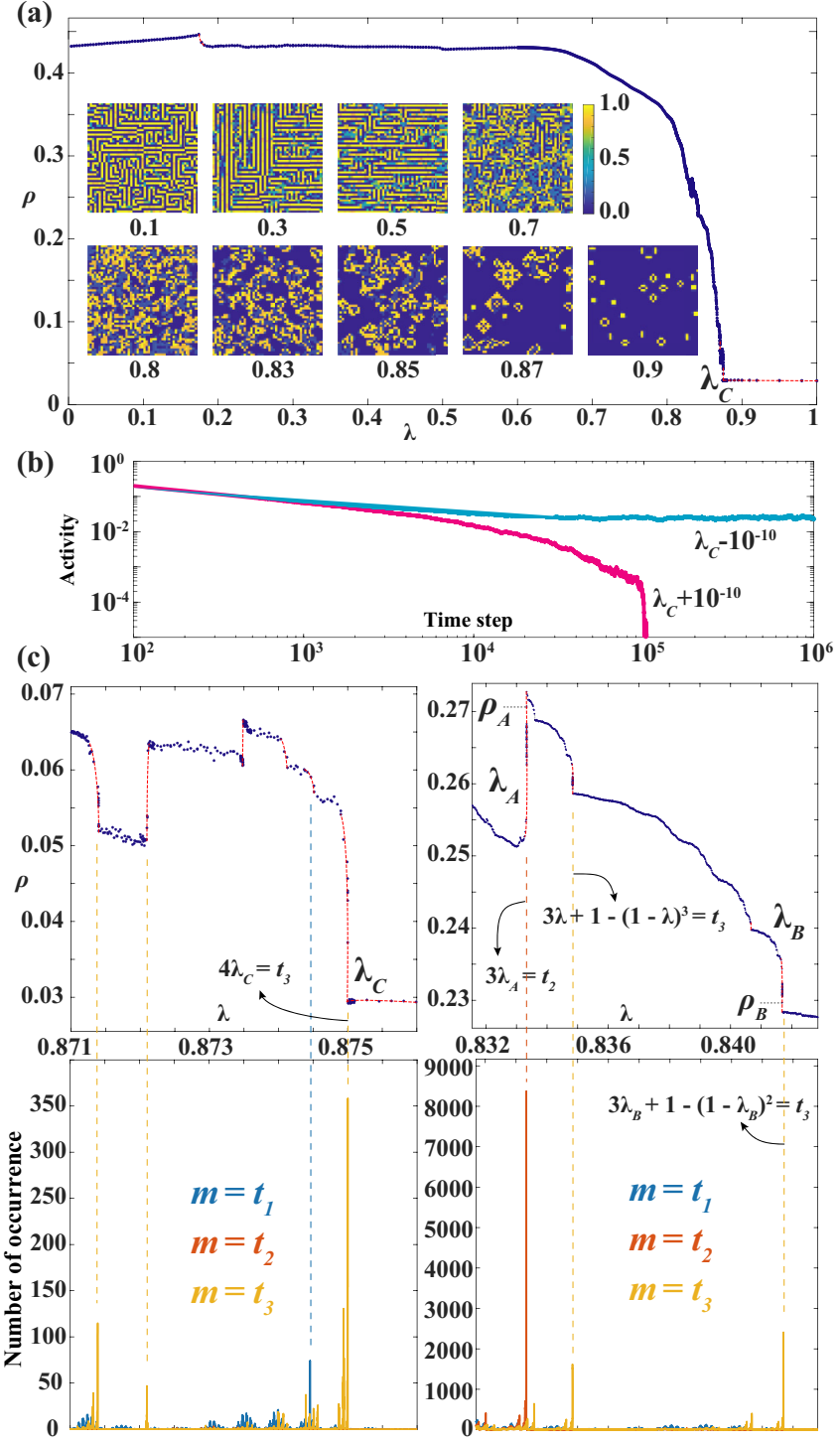

(d) $\times 10^{-3}$
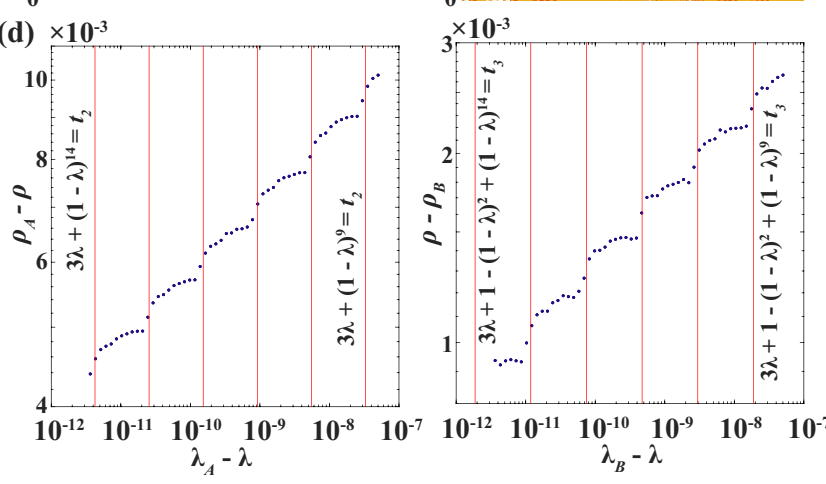

FIG. 3. (a) The asymptotic density, $\rho$, in the Logistic GOL with respect to the tuning parameter $\lambda$. Insets show snapshots of randomly chosen regions from the asymptotic state for different values of $\lambda$. The blue dots correspond to numerical results while the red dashed lines are a guide for the eye. (b) Log-log plot of the average diffusive activity (see the text) vs time for $\lambda$ slightly above (magenta) and below (blue) the threshold. (c) Top panel: two magnified regions of the plot in part (a). Bottom panel: the number of times the neighborhood sum $m$ is equal to either $t_{1}, t_{2}$, or $t_{3}$ with respect to $\lambda$. (d) Log-log plot of the transition at $\lambda_{A}$ and $\lambda_{B}$ present in (c). The red vertical lines delineate microtransitions (see the text). the remaining space keep decaying as their neighborhood mostly consists of six dense sites. In this way, the sites get correlated and help maintain larger-scale stable domains of such patterns.

The Cantor set that defines the state space becomes simply connected at $\lambda=0.5$, which is accompanied by a sudden change in the average density. As $\lambda$ approaches $\sim 0.18$, the flickers become isolated and the system makes a transition to an asymptotic state that has very low activity. In fact, the density of this asymptotic state depends on the initial conditions. Starting with a uniform distribution results in a linear dependence of $\rho$ on $\lambda$.

To further clarify the transition at $\lambda_{C}$, we calculate the timedependent diffusive activity of the system for $\lambda=\lambda_{C}+10^{-10}$ and $\lambda=\lambda_{C}-10^{-10}$. Here the activity at time step $t$ is calculated by first taking the difference of states at each cell by their corresponding values 60 time steps before and then finding the average of the absolute values of these differences. In this way, we probe only the activity that can propagate throughout the lattice, and we also obtain zero average activity when the system reaches the inactive asymptotic state (with oscillators having periods that are divisors of 60 ). All of the 28 runs reach an inactive asymptotic state at approximately $10^{5}$ steps when $\lambda=\lambda_{C}+10^{-10}$ while all of the 28 runs remain active after $10^{6}$ steps when $\lambda=\lambda_{C}-10^{-10}$. Logarithmic plots of average diffusive activities are presented in Fig. 3(b). At transient times one can observe the power-law behavior of diffusive activity. Then the slope of the line decreases and activity drops to zero for $\lambda_{C}^{+}$. However, it stabilizes to a finite value for $\lambda_{C}^{-}$. This clearly shows that $\lambda_{C}$ marks the point of sudden departure from the subcritical nature of Conway's GOL to an active asymptotic behavior.

In Fig. 3(c) we present a close inspection around $\lambda_{C}=$ 0.875 , which reveals that there are many other transitions that are present in $\lambda<\lambda_{C}$. To understand the source of the transition at $\lambda_{C}=0.875$ and the subsequent transitions, we must focus on conditions that change the operation regimes of the system. As the tuning parameter alters the rate of change in states, the neighborhood sums $m$ made up of these states are also tuned. Changes in operation regions occur when $m$ sums cross any of the limits $t_{1}, t_{2}$, or $t_{3}$. For example, if $m$ is slightly less than $t_{3}$, then the corresponding state will grow; however, if, upon tuning $\lambda$, the same sum becomes a value slightly larger than $t_{3}$, the site will decay. Hence, $m=$ $t_{3}$ will correspond to a transition between two significantly different pathways in the system. The more these "equalities" occur, the greater will be the impact on the global dynamics. To numerically investigate the values of $\lambda$ where significant changes are expected, we start from a $1000 \times 1000$ uniform initial state and run the system for 1000 steps. Then we run the system for another 1000 steps while recording the number of occurrences where $m$ is equal to $t_{1}, t_{2}$, or $t_{3}$ up to a precision $10^{-6}$ that is also the increment with which $\lambda$ is tuned. The number of occurrences versus $\lambda$ is plotted at the bottom panels of Fig. 3(c). As we expected, there is indeed a sharp peak at $\lambda=\lambda_{C}$ in the number of occurrences of $m=t_{3}$. Also the other transitions are accompanied by relevant peaks.

Now we need to explain why $m=t_{3}$ has a peak at $\lambda=\lambda_{C}$. We start by restating that at these values of $\lambda$, the system is dominated by the quiescent phase (regions of zeros) with 
sparse activity. States having values $\lambda$ keep forming as a result of a single growth operation on zeros at the boundaries where active sites meet the quiescent phase. Indeed, states involving fewer operations are formed more frequently than those needing more operations. In other words, states having a lower polynomial degree on $\lambda$ are more frequent than others. Hence the transition at $\lambda_{C}=0.875$ can be explained by $4 \lambda_{C}=t_{3}$, which corresponds to a quite frequent neighborhood populated by four $\lambda$ states and four zeros. As $\lambda$ is tuned down, the operation of this sum changes from decay to growth, and any cell with such a neighborhood starts growing instead of decaying, thereby altering the dynamics.

Another substantial change occurs at $\lambda_{A}=5 / 6$ when $m=$ $3 \lambda_{A}=t_{2}$ (a neighborhood of three $\lambda$ states and five zeros), as seen in Fig. 3(c). Here one of the frequently occurring sums changes its region from growth to stability and leads to a sudden decrease in density as $\lambda$ is decreased. This transition is different from the one at $\lambda_{C}$ as the system has active asymptotic states both before and after. Another related transition is present at $\lambda_{B} \sim 0.842$, which can be understood by employing the second-order values of the Cantor set, namely $\left\{(1-\lambda)^{2}, \lambda(1-\lambda), \lambda+(1-\lambda)^{2}, 1-(1-\lambda)^{2}\right\}$. Then the corresponding equation is $3 \lambda_{B}+1-\left(1-\lambda_{B}\right)^{2}=$ $t_{3}$. This transition in turn can be related to yet another transition at $\lambda \sim 0.834$ in which the third-order values are employed in the equation $3 \lambda+1-(1-\lambda)^{3}=t_{3}$. The magnitude of change in the density decreases as the sums $m$ involve more and/or higher-order terms and hence occur less frequently. Taking this idea further, one can claim that every transition point is accompanied by infinitely many nearby transition points involving ever less frequently occurring higher-order terms of the Cantor set. This claim is supported by the log-log plots of changes in the density versus changes in $\lambda$ near the critical points $\lambda_{A}$ and $\lambda_{B}$ presented in Fig. 3(d). Here, adding the smallest nonzero element of the $n$ th-order Cantor set, $(1-\lambda)^{n}$, to the original equations results in new equations corresponding to accompanying microtransitions shown by red vertical lines. Each deterministic transition follows a linear trend in the logarithmic scale, which is similar to the power-law behavior of critical transitions in stochastic systems, while the presence of cascadelike microtransitions yields a qualitatively different self-similar nature.

\section{AUTOCATALYTIC INTERACTIONS}

To understand how self-organized structures arise in the Logistic GOL, we formulate the tuning parameter range where propagators adaptively survive by investigating their local interactions. Propagators are actually the best indicators of self-organization in CA $[22,23]$. We first consider the Glider, an emergent propagator transmitting activity in the long range and also the key component in computational properties of Conway's GOL $[9,24]$. Glider is translated one diagonal cell every four steps.

In our model, it preserves its direction, speed, and periodicity in a continuous range of $\lambda$. As seen in Fig. 3(a), for $\lambda=0.8$ Glider has a similar structure, yet with different numerical values due to the altered updating rate. Note that
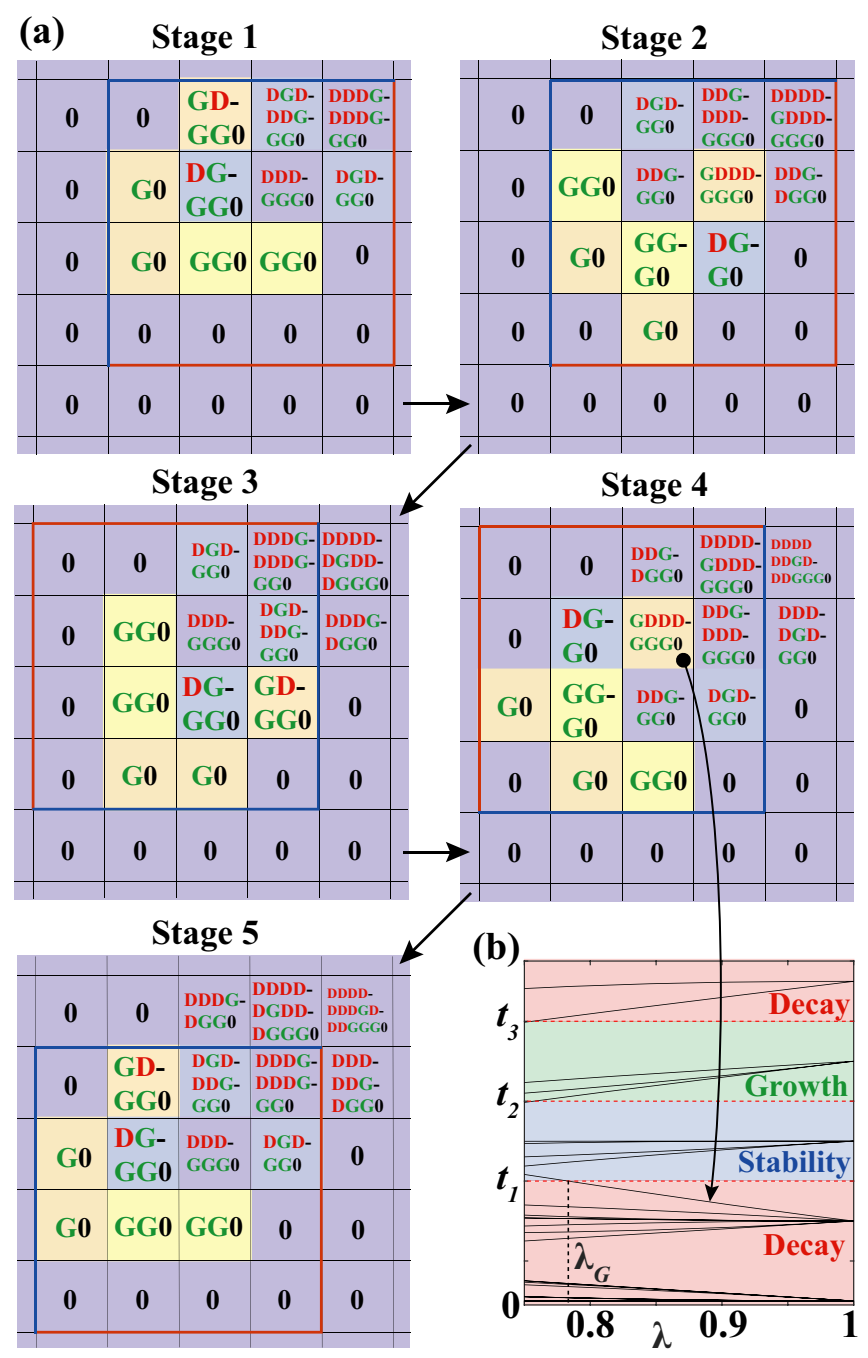

FIG. 4. (a) Five stages of evolution of the Glider in the Logistic GOL for $\lambda=0.8$. The transposes of the states delineated by red and blue contours are identical within even and odd stages. The set of nontrivial operations is preserved for the initial and final stages. (b) Neighborhood sums vs $\lambda$. The first change in the operation regions of sums occurs at $\lambda=\lambda_{G}$, which corresponds to the cell shown by the arrow.

behind any stable, oscillating, or propagating structure, there is a pertaining finite set of local interactions involved. We quantify these interactions by investigating the corresponding neighborhood sums $m$ under the operations employed by the rules. Considering the polynomial representation of states (operator notation introduced in Sec. II) is essential for this study because it allows us to extract quantitative information from the emerging propagators.

To find the parameter range in which the Glider can be preserved, we record the operations on each cell for every time step as this structure propagates in a field of quiescent states. This analysis is presented in Fig. 4(a) with stability operations omitted. Each decay or growth operation increases the polynomial order of the state by one degree. The $m$ for each site can thus be expressed in terms of $\lambda$-dependent polynomials. Once Glider leaves a certain region, the 
visited sites will keep decaying indefinitely due to exposure (underpopulation) and the neighborhood sums of these cells trivially fall in the decay region $(m \approx 0)$. Active cells, which lead the propagation mechanism, make up a finite set of recurring nontrivial operations corresponding to this structure, equivalent to attractors in dynamical systems [25]. Due to their self-generating character, we refer to these operations as autocatalytic interaction sets. As $\lambda$ is tuned, if these outertotalistic sums remain within their corresponding regions of operation, it means that the supposed operations will keep recurring, hence the Glider can still propagate under such growth and decay rates. Its structure will be altered when the first sum changes its operation region.

As seen in Fig. 4(b), the first sum that changes the dynamics and breaks this emergent behavior occurs at $\lambda_{G} \sim$ 0.783 , where a polynomial of 11 th order in $\lambda$ crosses $t_{1}$ from decay to stability. The order of this polynomial is dictated by DDDDGDDDGGG0, which is the highest-order state in Moore's neighborhood of the cell changing its operation regime. Notice that the presence of symmetries in this framework reduces the number of unique interactions significantly. These symmetries are highlighted by the red and blue contours in Fig. 4(a).

\section{RAYFISH: AN EMERGENT PROPAGATOR}

By tuning the parameter, we further notice the step-by-step imminent self-organization of a propagator that is only partially present in Conway's GOL. This period-36 orthogonal propagator, named Rayfish after its shape and dynamics [see Fig. 5(a)], appears in a short yet continuous range $\lambda_{A}<\lambda<$ $\lambda_{R}$. Here, $\lambda_{A}<\lambda$ supports the main propagation mechanism of the Rayfish, where a column of three $\lambda$ states gives birth to another $\lambda$ next to them. On the other limit, $\lambda_{R} \sim 0.841$ is the solution to another 11th-order polynomial equation in $\lambda$, calculated using the method presented in Sec. IV. A total of 22 of the 36 links in the Rayfish loop are also present in Conway's GOL, including the six consecutive steps (from 11 to 17) that connect the largest stages. These stages are easily noticed in active regions of Conway's GOL. On the other hand, stages from 24 to 29 are very simple structures that are easily reached, making Rayfish a very frequent attractor that contributes to the large increase in density at $\lambda_{A}$ as $\lambda$ is increased.

In Fig. 5(b), using the recorded neighborhood sums of Rayfish (similarly as with the Glider), the supported stages are shown as $\lambda$ is varied. The structure is set only once to propagate in a field of empty states, and, by recording the operations, we can extrapolate information about every stage of Rayfish in the whole parameter range. Having obtained the results, from a reverse perspective one can see how tuning $\lambda$ down gets the neighborhood sums coupled step by step until they reach the autocatalytic regime, where all the stages are supported and there is no interaction that breaks the loop. Outside the range $\lambda_{A}<\lambda<\lambda_{R}$, stages start breaking down, making this attractor not reachable anymore, while the number of neighborhood sums changing operation regions increases and further deteriorates the loop. Thus, it is important to emphasize not only the robustness and inevitability of this propagator inside its operating range, but also the vulnera-
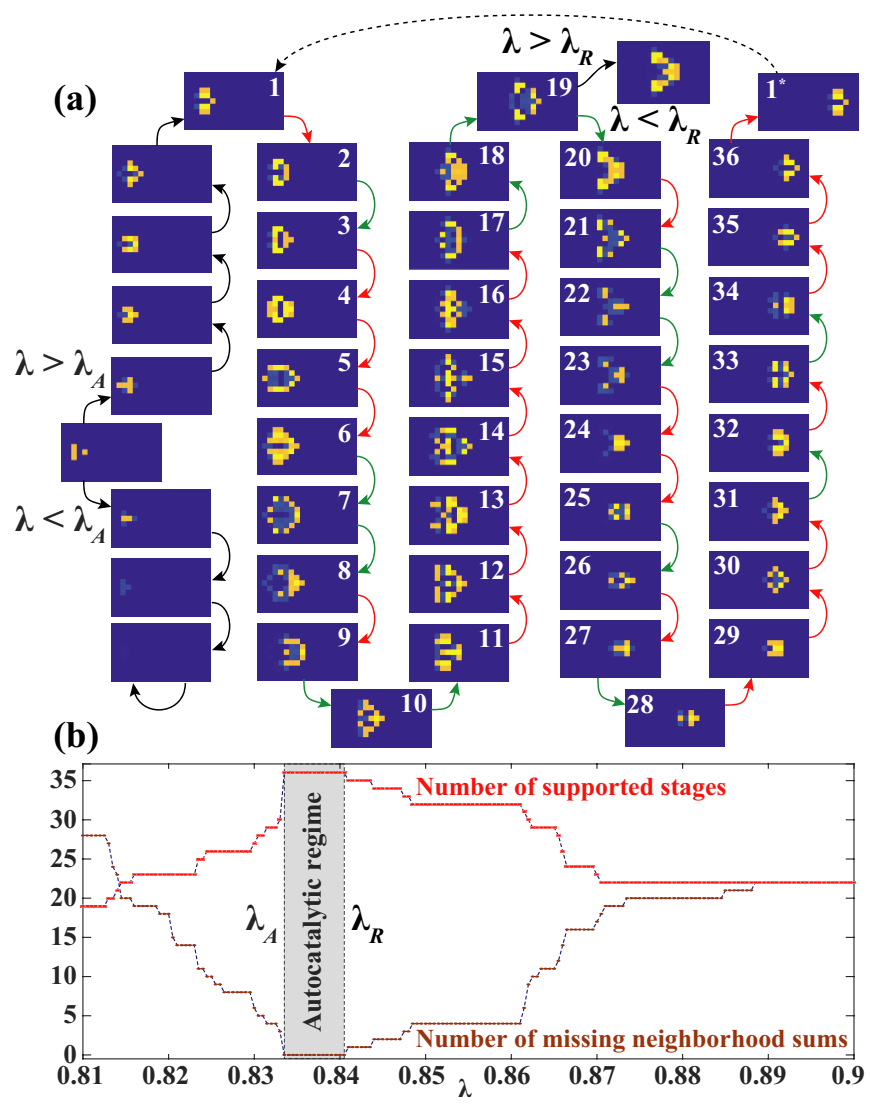

FIG. 5. (a) Formation of the period-36 propagator named Rayfish from simple initial conditions. The red arrows indicate the steps present also in Conway's GOL. $1^{*}$ and 1 denote the same but translated states. (b) Red lines represent the number of Rayfish stages that are dynamically reachable vs $\lambda$. The stages supported by the neighborhood sums form an autocatalytic loop only in the shaded interval. Outside this interval, the neighborhood sums change their operation regions, as shown by the brown lines.

bility and its incomplete unveiling outside that range. Being quite abundant while possessing sheer complexity are two properties of this emergent system that, instead of remaining mutually exclusive, counterintuitively start supporting each other.

The development process itself and properties of Rayfish make it a suitable abstract model for investigating the origins of life scenarios based on self-organizing autocatalytic reaction sets [26]. In the context of CA, Rayfish is an epitomic example of how local sites get coupled in a consequent series of interactions that generate a self-sustained loop. There is a spatiotemporal synchronization of values and operations, which effectively exploits the different symmetries of the framework, thereby introducing consonance in a higher hierarchy even though only local interactions are involved [27].

\section{THE LOGISTIC RULE 90}

Our framework can be embodied in other outer-totalistic CA as well. In particular, we study Rule 90, a one-dimensional elementary CA in which the next value of a cell is determined by the sum of its two nearest neighbors [28]. The actions 
(a)

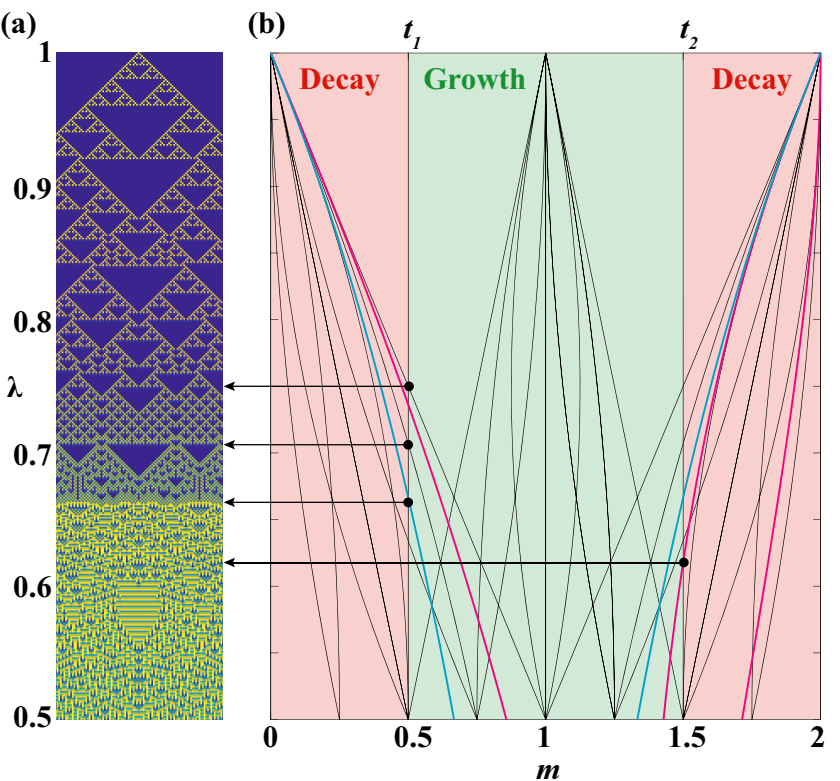

(c)

(d)

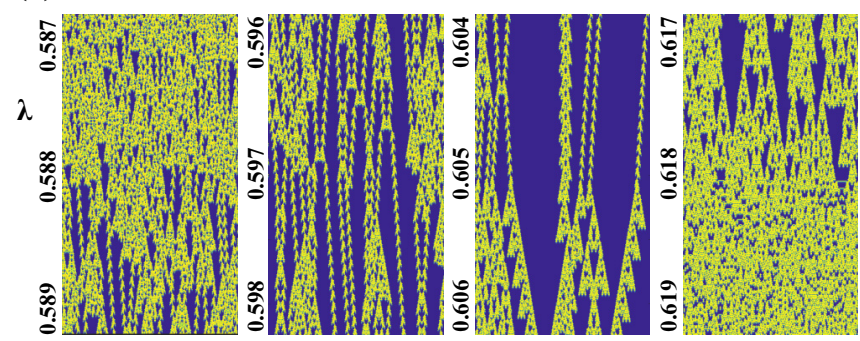

FIG. 6. (a) Time evolution of Logistic Rule 90 accompanied by a decrease in $\lambda$. (b) Thin black lines represent all unique sums that can be constructed by adding to elements from the second-order Cantor set. The cyan lines represent the $2 s_{1}$ and $2 s_{2}$ sums of period-2 stripes (see the text). The magenta lines represent the $2 s_{1}, 2 s_{2}$, and $2 s_{3}$ sums of period-3 stripes. Arrows show the important changes in dynamics to crossings of sums between decay and growth operations. (c) Transformation that maps period-3 stripes into fields of zeros for better visualization. (d) Four snapshots of larger implementation of the same system whereby in 10000 steps $\lambda$ is increased from 0.575 to 0.625 , starting with 2500 cells having random initial values and periodic boundary conditions.

correspond to decay and growth if the modulo 2 of the sums are 0 and 1 , respectively. Introducing $\lambda$ transforms the neighborhood sum into a continuous set in the range $[0,2]$ populated by twofold convolution of the $\lambda$-dependent Cantor set. We assign the growth interval to the range $[0.5,1.5]$ sandwiched between two decay regions. In Fig. 6(a), we show how the dynamics of the logistic version of Rule 90 changes when $\lambda$ is decreased as the system starts from a single nonquiescent cell. Even though $\lambda$ is tuned while the system is run, initially the system evolves in the same fashion with the original Rule 90 producing the fractal object called Sierpinski triangle.

At $\lambda=0.75$, the dynamics of the system changes substantially for the first time. To understand this change, we plot all unique sums produced by adding two elements from the second-order Cantor set assuming that these are the most frequently occurring ones. Any of the sums changing operation region as $\lambda$ is varied could induce different dynamics in the system. As seen in Fig. 6(b), at $\lambda=0.75$ two of the sums cross $t_{1}$ and $t_{2}$ lines, both going from decay to growth. The crossing sums correspond to formulas $2(1-\lambda)=t_{1}$ and $2 \lambda=t_{2}$. In fact, all crossings come in symmetric pairs because of the symmetry in both the Cantor set and the rules of this particular system. The next crossing occurs when the sum of the elements $(1-\lambda)$ and $(1-\lambda)-(1-\lambda)^{2}$ is equal to $t_{1}$, which corresponds to $\lambda=1 / \sqrt{2}$. This point is also accompanied by a substantial change in dynamics. The arrows extended from the transition points [see Figs. 6(a) and 6(b)] also coincide with different regimes of textures emerging in the system.

The next significant change in dynamics, however, occurs for a different reason. To understand this, we need to consider the possibility of having oscillating states. The simplest case would be having states with a value $s_{1}$, dominating a region of space, altogether changing their values to $s_{2}$ and then returning back to $s_{1}$ and repeating this oscillatory behavior. Assuming that $s_{2}>s_{1}$, these kinds of period-2 stripes (in time domain) are supported if $\mathbf{G} s_{1}=s_{2}$ and $\mathbf{D} s_{2}=s_{1}$. Solving these equations simultaneously, one gets $s_{1}=(1-\lambda) /(2-$ $\lambda)$ and $s_{2}=1 /(1-\lambda)$. However, to be in the correct operation regime, we also need to have $2 s_{1}>t_{1}$ and $2 s_{2}>t_{2}$. The sums $2 s_{1}$ and $2 s_{2}$ are plotted with cyan lines in Fig. 6(b). One can see that $2 s_{1}>t_{1}$ holds for $\lambda<2 / 3$ while $2 s_{2}>t_{2}$ holds for $\lambda>2 / 3$. This makes $\lambda=2 / 3$ a very special point where period- 2 stripes are supported. Indeed, running the system with $\lambda=2 / 3$ (not shown here) results in complicated dynamics dominated by period- 2 stripes. The same arguments apply for a checkerboard pattern but this time with $2 s_{1}<t_{1}$ and $2 s_{2}<t_{2}$. One can spot both the checkerboard pattern and period-2 stripes in the vicinity of $\lambda=2 / 3$ but they quickly fade because they lack autocatalytic sets as described above. The cyan lines in Fig. 6(b) representing the sums that support these patterns result in decay for $\lambda<2 / 3$ and growth for $\lambda>2 / 3$. Hence, the average density increases as one passes over $2 / 3$ while decreasing $\lambda$.

Decreasing $\lambda$ further, we hit a point where period3 stripes [29] emerge and start dominating the system. One way of getting a period-3 stripe is to have $\mathbf{G} s_{1}=$ $s_{2}, \mathbf{G} s_{2}=s_{3}$, and $\mathbf{D} s_{3}=s_{1}$. Solving these equations simultaneously, one gets $s_{1}=(1-\lambda)(2-\lambda) /\left(\lambda^{2}-3 \lambda+3\right)$, $s_{2}=\left[(2-\lambda) /\left(\lambda^{2}-3 \lambda+3\right)-\lambda\right] /(1-\lambda)$, and $s_{3}=(2-$ $\lambda) /\left(\lambda^{2}-3 \lambda+3\right)$. Being in the correct operation regime requires conditions $2 s_{1}>t_{1}, 2 s_{2}<t_{2}$, and $2 s_{3}>t_{2}$. The sums $2 s_{1}, 2 s_{2}$, and $2 s_{3}$ are plotted with magenta lines in Fig. 6(b). All conditions are satisfied starting with the point where $2 s_{2}=t_{2}$, which corresponds to the equation $\lambda^{3}-2 \lambda+1=$ 0 , which has a root at $\lambda=(\sqrt{5}-1) / 2$ (the golden ratio). As seen in Fig. 6(b), from this point on period-3 stripes indeed appear and start dominating the system as $\lambda$ is tuned down. Note that the values $s_{1}, s_{2}$, and $s_{3}$ are not members of any low-order Cantor set. In fact, their autocatalytic nature forces the system to approach these values using higher orders of the Cantor set. In other words, very high-order elements of 
the Cantor set that are normally extremely rare get boosted to the point where they start to prevail over the system dynamics. This is another excellent example of emergent behavior.

At $\lambda \sim 0.6$, the period-3 stripes reach their peak size while localizing chaotic regions up to a point where the latter become propagators interacting with each other in a complex fashion. These propagators become more apparent after a transformation introduced in Fig. 6(c). Here we make use of the fact that $s_{1}+s_{2}+s_{3}=2$. Then instead of plotting $s^{t}$, which corresponds to the state at time step $t$, we plot $\mid s^{t-1}+$ $s^{t}+s^{t+1}-2 \mid$, in which case stripes appear as homogeneous fields of zeros. To reveal the rich dynamics around $\lambda \sim 0.6$, we start with 2500 cells having uniform distribution between $[0,1]$. Then in 10000 steps we increase $\lambda$ from 0.575 to 0.625 . In Fig. 6(d) we present four small snapshots of this system. One can see that around $\lambda \sim 0.587$, period- 3 stripes (blue regions) have no dominance. This is because the gap between the values $s_{1}, s_{2}, s_{3}$ and the Cantor set elements that are closest to them is too wide to be bridged with autocatalysis. As $\lambda$ is increased, period-3 stripes smoothly grow in size while propagators start appearing. Hence, in the context of canonical classification of CA [2], we observe a transition from "chaotic" Class 3 phase to complex "lifelike" Class 4 phase. This kind of transition was investigated earlier in the context of "computation at the edge of chaos" [19]. The advantage of our system is that we achieve the same transition while tuning a single parameter continuously.

The range $0.595<\lambda<0.605$ is dominated with a period18 propagator that moves in the field of period-3 stripes. The autocatalytic interactions of this propagator are more complicated compared to those of Rayfish, which propagates in the field of zeros. $\lambda \sim 0.605$ marks an unprecedented case in which the autocatalytic interaction set of period-18 propagator is broken while a different set supporting a new period-48 propagator is formed. This resonates with the idea of punctuated equilibrium where an organism acquires a substantial change in a short period of time to adapt to a new fitness landscape [30,31]. The period-48 propagator dominates the system dynamics until we reach $\lambda=(\sqrt{5}-1) / 2$ where they disappear since the system stops supporting period-3 stripes that act as their habitat.

\section{DISCUSSION}

In this study, aiming to reemphasize the importance of CA as proper tools for complex systems, we report a simple implementation of a decay and growth rate tuning parameter that can be extended to any dimensional two-state outer totalistic model. Applying this parameter to Conway's GOL brings up a transformation of the state space into a Cantor set, and a series of deterministic transitions with the emergence of self-organized patterns. Furthermore, we discuss how the same system can serve as a playground for the emergence of propagating spatiotemporal correlations under the influence of a single parameter. We also show that the same idea can induce similar behavior even in a one-dimensional system such as Rule 90. Next to the Logistic GOL, just by changing the stability and/or growth intervals $\left(t_{1}, t_{2}, t_{3}\right)$ one can generate all the possible birth/survival rules of other
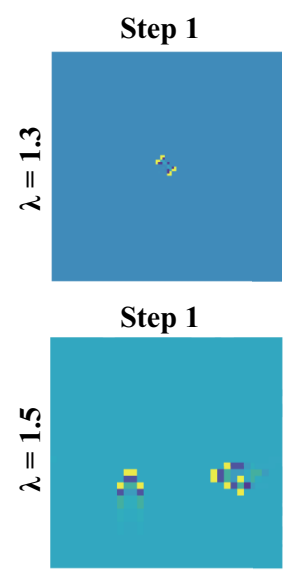

Step 1

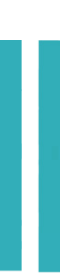

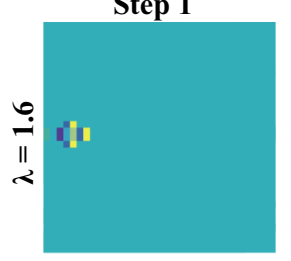

Step 13

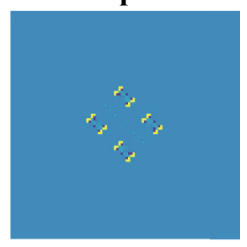

Step 10

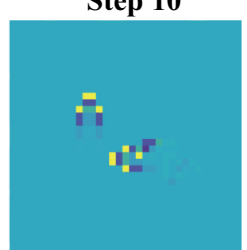

Step 12

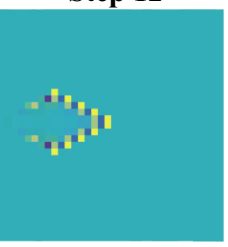

Step 40

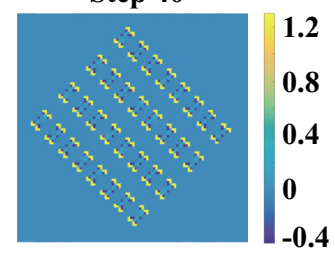

Step 18

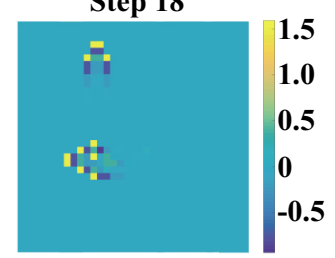

Step 23

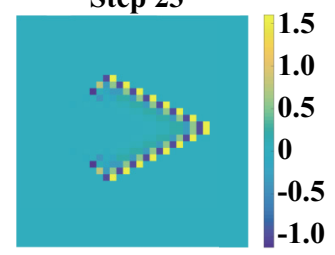

FIG. 7. Snapshots showing simple self-replicating structure (top) at $\lambda=1.3$, propagating ships at $\lambda=1.5$ (middle), and another structure that instantaneously self-replicates and propagates throughout the lattice at $\lambda=1.6$ (bottom).

lifelike CA, and exploit the approaches followed in this work, to explore novel examples of this discrete complex realm.

A further examination would lead to a plethora of patterns, propagators, and other self-organizing entities that appear under different rules (examples can be found in Ref. [32]), and all these can serve as phenomenological assets to provide helpful insights into different physical phenomena [33]. Interestingly, the Logistic GOL presents additional phenomena when operating with faster dynamics, namely in the $\lambda>1$ regime. Complex features emerge in the range $1.25<\lambda<1.6$ (see Fig. 7), where along with a burst of many different propagators, different self-replicating topologies appear. Each of these examples is still relying on finite recurring sets of interactions, but they exploit the symmetries of the framework in different levels.

Our results will shed light on parameter tuned class transitions and phase transitions in deterministic CA, questions addressed earlier [34] but never with a parameter that can be varied continuously. On the other hand, it is also plausible to consider this work as an extension of totalistic CA to coupled map lattices. The transition in Logistic GOL is qualitatively similar to chaotic transitions in coupled map lattices exhibiting spatiotemporal chaos [35]. Furthermore, the analysis of propagators as emergent phenomena, and their development, adaptation, or transformation over continuous ranges of parameters, is another focal point that contributes to an understanding of autocatalytic loops in real chemical or biological systems and how they are affected by possible changes in exterior conditions. 


\section{ACKNOWLEDGMENTS}

We thank M. Cemal Yalabik, H. Rouault, M. Özgür Oktel, A. Kerimov, and S. Ilday for helpful discussions, and $\mathrm{M}$. Yaman, A. Hejazy, and G. Dalipaj for a critical reading of the manuscript. S.J. acknowledges support from The Turkish Academy of Sciences-Outstanding Young Scientists Award Program (TÜBA-GEBIP). Part of the computational resources is provided by the National Center for High Performance Computing of Turkey (UHeM) under Grant No. 5003622015.
[1] J. von Neumann, Theory of Self-Reproducing Automata, edited and completed by A. W. Burks (University of Illinois Press, Urbana, IL, 1966).

[2] S. Wolfram, Nature (London) 311, 419 (1984).

[3] U. Frisch, B. Hasslacher, and Y. Pomeau, Phys. Rev. Lett. 56, 1505 (1986)

[4] C. Saloma, G. Jane Perez, G. Tapang, M. Lim, and C. PalmesSaloma, Proc. Natl. Acad. Sci. USA 100, 11947 (2003).

[5] R. White and G. Engelen, Environ. Plan. A Econ. Sp. 25, 1175 (2006).

[6] H. Seybold, J. S. Andrade, Jr., and H. J. Herrmann, Proc. Natl. Acad. Sci. USA 104, 16804 (2007).

[7] A. Deutsch and S. Dormann, Cellular Automaton Modeling of Biological Pattern Formation (Birkhäuser, Boston, 2005).

[8] L. Manukyan, S. A. Montandon, A. Fofonjka, S. Smirnov, and M. C. Milinkovitch, Nature (London) 544, 173 (2017).

[9] F. Bagnoli, R. Rechtman, and S. Ruffo, Phys. A 171, 249 (1991).

[10] L. S. Schulman and P. E. Seiden, J. Stat. Phys. 19, 293 (1978).

[11] D. Eppstein, Game of Life Cellular Automata, edited by A. Adamatzky (Springer, New York, 2010), pp. 71-97.

[12] K. M. Evans, Physica D 183, 45 (2003).

[13] S. Y. Huang, X. W. Zou, Z. J. Tan, and Z. Z. Jin, Phys. Rev. E 67, 026107 (2003).

[14] J. Nordfalk and P. Alstrøm, Phys. Rev. E 54, R1025(R) (1996).

[15] H. J. Blok and B. Bergersen, Phys. Rev. E 59, 3876 (1999).

[16] S. M. Reia and O. Kinouchi, Phys. Rev. E 89, 052123 (2014).

[17] P. Bak, K. Chen, and M. Creutz, Nature (London) 342, 780 (1989).

[18] R. M. May, Nature (London) 261, 459 (1976).

[19] C. G. Langton, Physica D 42, 12 (1990).
[20] K. Kaneko, Chaos 2, 279 (1992).

[21] H. Chaté and P. Manneville, J. Stat. Phys. 56, 357 (1989).

[22] S. Wolfram, Rev. Mod. Phys. 55, 601 (1983).

[23] S. Wolfram, A New Kind of Science (Wolfram Media, Champaign, IL, 2002).

[24] P. Rendell, in Designing Beauty: The Art of Cellular Automata, edited by A. Adamatzky and G. J. Martnez (Springer International, Cham, 2016), pp. 149-154.

[25] S. H. Strogatz, Nonlinear Dynamics and Chaos: With Applications to Physics, Biology, Chemistry, and Engineering, Studies in Nonlinearity (Westview, Perseus Books Group, Cambridge, 2001).

[26] S. A. Kauffman, The Origins of Order: Self-Organization and Selection in Evolution (Oxford University Press, New York, 1993).

[27] E. F. Codd, Cellular Automata (Academic Press, New York, 1968).

[28] O. Martin, A. M. Odlyzko, and S. Wolfram, Commun. Math. Phys. 93, 219 (1984).

[29] T. Y. Li and J. A. Yorke, Am. Math. Mon. 82, 985 (1975).

[30] N. Eldredge and S. J. Gould, Punctuated equilibria: An alternative to phyletic gradualism, in Models in Paleobiology, edited by T. J. M. Schopf (Freeman, San Francisco, 1972), pp. 82-115.

[31] P. Bak and K. Sneppen, Phys. Rev. Lett. 71, 4083 (1993).

[32] M. Ibrahimi, M.S. thesis, Bilkent University, 2019.

[33] B. Chopard, in Computational Complexity: Theory, Techniques, and Applications, edited by R. Meyers (Springer, New York, 2012), pp. 407-433.

[34] W. K. Wootters and C. G. Langton, Physca D 45, 95 (1990).

[35] T. Bohr, M. van Hecke, R. Mikkelsen, and M. Ipsen, Phys. Rev. Lett. 86, 5482 (2001). 